DOI: 10.17148/IARJSET.2021.8886

\title{
Cuffless, Non-invasive Blood Pressure Monitoring using ECG and PPG
}

\author{
Ms. Shreelakshmi $\mathbf{P}^{1}$, Mrs. Rajeswari $\mathbf{P}^{2}$ \\ ${ }^{1}$ PG Research Scholar - Electronics \& Instrumentation Engineering, JSS Science \& Technology University, Mysuru, \\ Karnataka \\ ${ }^{2}$ Assistant Professor, Electronics \& Instrumentation Engineering, JSS Science \& Technology University, Mysuru, \\ Karnataka
}

\begin{abstract}
Blood pressure (BP) is one the very important and vital parameter in clinical healthcare. Nearly approx. 8 million deaths happen due to high blood pressure. So, it is important to monitor blood pressure every now and then. For prolonged years, for measurement of blood pressure cuff based sphygmomanometer has been one of the gold standard method. Later, cuff-based arterial invasive line has also been used for blood pressure measurement under physician care to access BP. In the recent few years, cuff-based oscillometry is used for BP measurement at home healthcare and remote healthcare which is more convenient and easily accessible. But, it has few setbacks namely - inaccurate results due to movement, discontinuous nature, cuff based is painful, unable to integrate it with mobile application and calibration. Recently, many studies and approach to get BP values using various methods are deeply investigated, where BP values can be obtained through time delay in the pressure wave propagation in vascular system of body. Hence, it paved a way for cuffless, non-invasive blood pressure measurement for both patients and healthy persons. We present a wearable watch which is used to estimate Blood Pressure (BP) in real time with the help of ECG (Electrocardiogram) and PPG (Photoplethysmogram). Firstly get the ECG and PPG data from wrist using ECG electrodes and PPG sensor, which is attached to wearable watch. ECG is electrical activity of heart muscle and PPG is optical activity of heart muscle. Preprocess the ECG and PPG data using suitable filters like notch filter, bandpass filter to remove unwanted noises and cross correlate it. Detect the R peak of ECG and PPG peak using peak detection algorithm based on threshold. Obtain time delay between ECG R peak and PPG peak, which is Pulse Transit Time (PTT). Using ECG and PPG peaks Heartrate is calculated. Then, PTT and Heartrate are the input to model to give an estimation of Systolic BP and Diastolic BP. Challenge is to record ECG data and PPG data simultaneously and process the data, so that peaks are visible to get PTT and in turn to obtain blood pressure readings on wearable watch.
\end{abstract}

Keywords—Blood Pressure, ECG, PPG, PTT, Signal Processing, Peak Detection.

\section{INTRODUCTION}

Cardiovascular disease caused around 17 million deaths globally in 2020, accounting for 1/3rd of all deaths. This ratio is found to be increasing even in developing countries like India. So blood pressure monitoring is a significant prognostic factor for CVD (Cardio Vascular Disease) events. Clinically, ambulatory BP monitoring [invasive method] or auscultationor cuff based method is adopted. But these are not suitable for everyday observations.

$\mathrm{BP}$ is very important parameters to be known to get to know the persons overall health conditions. It consists of two important components, one of it is - systolic blood pressure (SBP) and other one is - diastolic blood pressure (DBP). BP of a person depends on various factors namely age of the person, heredity of the person, diet of the person, lifestyle of the person and profession of the person. It is also life threatening issue may be due to changes in life style and adaptation to western foods by recent generation.

In 2014, World health organization (WHO) given a report which stated approx. $40 \%$ of adult population from age 25 or more globally suffering from either hypertension or hypotension. Due to this, in the developing countries and for new 


\section{International Advanced Research Journal in Science, Engineering and Technology}

Vol. 8, Issue 8, August 2021

\section{DOI: $10.17148 /$ IARJSET.2021.8886}

lifestyle, one needs continuous monitoring of BP which is very essential for ones day to day life to avoid and prevent life threatening conditions like cardiovascular diseases, stroke, kidney failure, arterial narrowing, vertigo, dementia, fatigue, transient ischemic attack, dyspepsia and few more. Hence there is a demand for cuffless, non-invasive, continuous monitoring of BP which can be useful for remote health care, also easy to operate and use among people.

The aim of this paper is to measure the blood pressure of the person without using cuff and without invasive methods. It is very useful for home healthcare, for BP monitoring of different age group which is add on point for elderly persons, for physicians who doesn't have to be available all time to persons who have normal BP range. BP can lead to many serious cardiac related problems, if not treated and monitored.

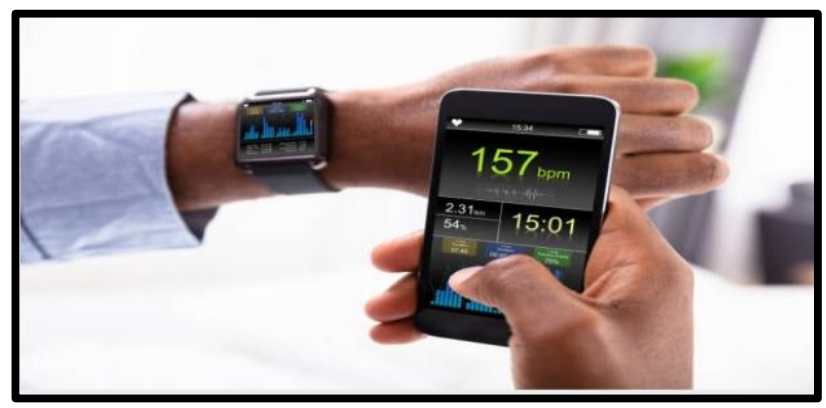

Fig 1. ECG and PPG in Wearables for BP Monitoring.

\section{LITERATURE REVIEW}

The methodologies used in this paper was developed after studying the various experiments conducted on cuff based and cuffless blood pressure monitoring. The following literatures are the works relevant to Cuffless, Noninvasive Blood Pressure monitoring with ECG and PPG

S. Weber proposed a measurement type in 2012 which was Non-invasive, Continuous with cuff that used ultrasound sensing method. This technique was based on frequency difference between transmitted and reflected ultrasound wave when passed through arteries. Advantage of this technique was automatic measurement, hence easy to use. Disadvantage of this techniques were complex measurement technique, trained practitioner were needed, device was quite costly and difficult to integrate with remote healthcare system. [10]

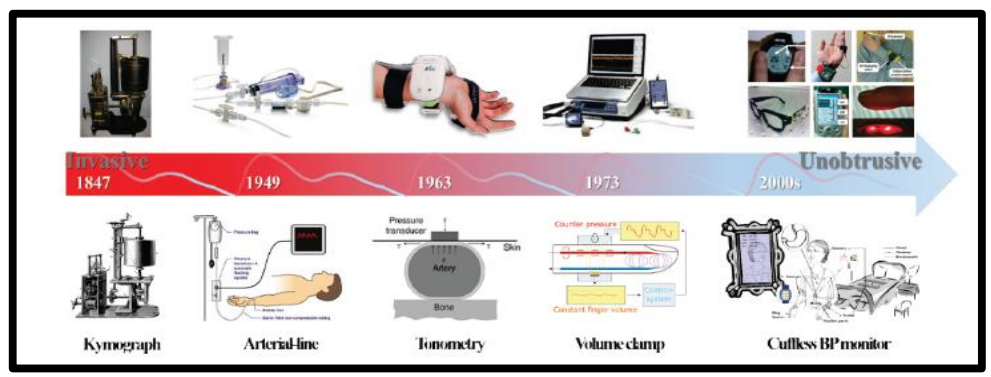

Fig 2. Timeline of Continuous Blood Pressure Monitoring Techniques

Agnes S. Meidert proposed a measurement type in 2014 which was Non-invasive, Continuous with cuff that used vascular unloading method. This technique was based on finger PPG sensor that adjusts pressure to keep optically measured finger vascular blood volume constant. Advantage of this technique were automatic measurement, hence easy to use and can be integrated with remote healthcare system. Disadvantage of this techniques were comparatively, the device was costly and finger PPG sensor output varies from subject to subject. Hence, device calibration was needed for each subject. [3] 
Vol. 8, Issue 8, August 2021

\section{DOI: 10.17148/IARJSET.2021.8886}

S. Noh proposed a measurement type in 2014-15 which was Non-invasive, Continuous with cuff that used tactile sensing method. This technique was based on flattening of the radial artery and measured pressure exile through skin. Advantage of this technique were automatic measurement, hence easy to use, portable and can be integrated with remote healthcare system. Disadvantage of this techniques were regular calibration needed as the measurement depends on subject's skin elasticity, curvature, location of sensor, etc. and uncomfortable for prolonged use. [9]

Timothy B. Plante proposed a measurement type in 2016-17 which was Non-invasive, Continuous with cuffless that used PPG with Near-Infrared method. This technique was based on change in blood vessel diameter in each cardiac cycle obtained by the NIR. Advantage of this technique were it was without cuff continuous noncontact measurement technique and can be integrated with remote healthcare system. Disadvantage of this techniques were measurement varies due to existence of surrounding NIR light sources and exact BP value measurement is not possible. [6]

Ramakrishna Mukkamala proposed a measurement type in 2015-16 which was Non-invasive, Continuous with cuffless that used pulse transit time using ECG and PPG senor on finger method. This technique was based on measuring the time difference between ECG signal \& Finger PPG Signal. Advantage of this technique were it was without cuff continuous measurement technique and can be integrated with remote healthcare system. Disadvantage of this techniques were comparatively

the device was costly and measuring devices are also introduced, some delay during time difference measurement, which leads to wrong interpretation of BP. [7]

\section{EXPERIMENTAL METHODS OR METHODOLOGY}

The three ECG electrodes and one PPG sensor are attached to wrist watch. ECG and PPG signal from wrist are collected and it is passed to ECG amplifier and PPG amplifier. Here, the low signal gets amplified. Then after that it is passed through hardware filters and filtered data are stored in ST microcontroller. When the data is read, it is fetched as raw ECG data and PPG data respectively. It is then passed through digital filters of ECG and PPG signal. Once the noisy raw ECG and PPG data are cleaned, it is passed through peak detection algorithm to find ECG and PPG peaks. Using ECG peaks and PPG peaks, ECG heartrate, PPG heartrate and PTT can be calculated. With the help of PTT, ECG heart rate and PPG heart rate, Blood pressure can be calculated. It is displayed on the watch and as well as on inbuilt application through bluetooth.

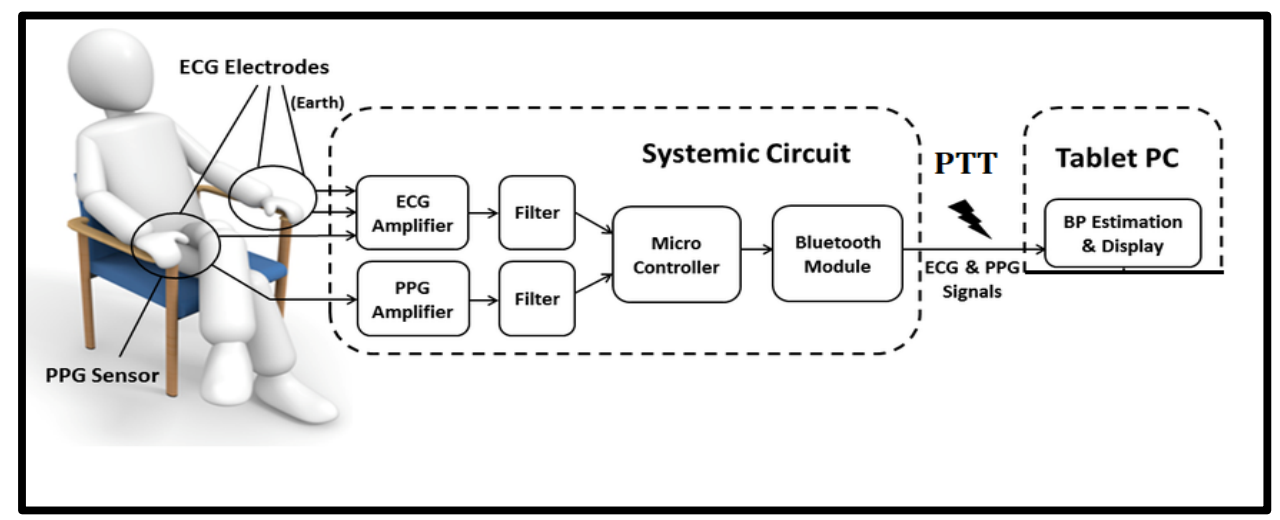

Fig 3. Block Diagram of Blood Pressure Estimation Using ECG And PPG.

\subsection{ECG and PPG Data Processing}

When raw ECG and PPG data is read, its ADC values are fetched and stored in an array. Raw ECG signals frequency band is between $0.05 \mathrm{~Hz}$ to $100 \mathrm{~Hz}$. Raw ECG data is passed through bandpass filter where it removes low and high frequency data which is between $0.05 \mathrm{~Hz}$ to $99 \mathrm{~Hz}$. It is further passed through notch filter to remove power line noise of 


\section{International Advanced Research Journal in Science, Engineering and Technology}

Vol. 8, Issue 8, August 2021

DOI: 10.17148/IARJSET.2021.8886

$50 \mathrm{~Hz}$. Then Next, it is passed through median filter to remove baseline drift. Further, it is passed to moving average filter to smoothen the signal. It is then passed to peak detection algorithm, here PQRST peaks are detected using peak detection algorithm. Using R peak location ECG heartrate is calculated and R peak locations are stored in an array. Clean ECG is displayed on inbuilt application.

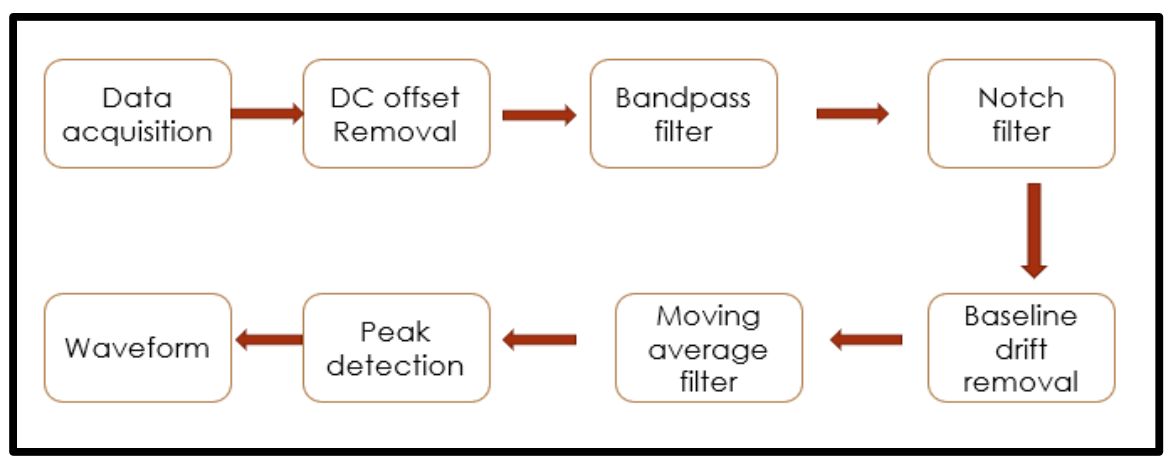

Fig 4. Data Processing of ECG and PPG

Raw PPG signal frequency band is between $0.05 \mathrm{~Hz}$ to $4 \mathrm{~Hz}$. Raw PPG data is passed through bandpass filter where it removes low and high frequency data which is between $0.05 \mathrm{~Hz}$ to $3.9 \mathrm{~Hz}$. It is further passed through notch filter to remove power line noise of $50 \mathrm{~Hz}$. Next, it is passed through median filter to remove baseline drift. Further, it is passed to moving average filter to smoothen the signal. It is then passed to peak detection algorithm, here peaks of PPG are detected using peak detection algorithm. Using PPG peak location PPG heartrate is calculated and PPG peak locations are stored in an array.

\subsection{PTT Calculation}

PTT is a pulse transit time, a time interval between the ECG peak area and the PPG peak. PTT is used to calculate Systolic blood pressure and Diastolic blood pressure.

$$
\begin{gathered}
\text { PTT }=\text { PPG Peak Distance }- \text { ECG Peak Distance } \\
\text { Average PTT distance }=\text { PTT } / \text { Number of PTT distance }
\end{gathered}
$$

In relation to PTT is equivalent to BP. That is, when there is too much BP, PTT is reduced and vice versa. PTT should be a good number. Calculate PTT for each ECG R and PPG value. Once the PTT is calculated for each value. Calculate the distance between PTT using a scale or by estimating the total PTT distance divided by the PTT range value.

\subsection{BP Calculation}

BP require PTT, ECG heartrate and PPG heartrate. Blood pressure mainly consists of two elements, one of which is systolic blood pressure, the highest pressure produced during the cardiovascular cycle. Another diastolic blood pressure, a small pressure created during the heart cycle.

$$
\begin{gathered}
\text { HRms }=(60000 / \text { ECG heartrate }) \\
\text { Td }=\text { HRms }- \text { Average PTT distance } \\
\text { Systolic pressure }=(\mathrm{A}-(\mathrm{B} * \text { ECG Heartrate })+(\mathrm{C} * \mathrm{Td})) * \mathrm{D} \\
\text { Diastolic pressure }=(\mathrm{E}-(\mathrm{F} * \mathrm{PPG} \text { Heartrate })+(\mathrm{G} * \mathrm{Td})) * \mathrm{H}
\end{gathered}
$$

In the above equation, HRms is Heartrate in ms. Td is Time delay. A, B, C, D are Systolic factors. E, F, G, H are Diastolic factors. 
Vol. 8, Issue 8, August 2021

\section{DOI: $10.17148 / I A R J S E T .2021 .8886$}

\section{RESULTS AND DISCUSSION}

The main aim of this paper is to get blood pressure using ECG and PPG without Cuff and Non-invasively. To achieve blood pressure in wrist wearable smart watch, We need to collect the ECG data and PPG data from ECG and PPG sensors tied on the wrist. After collecting the ECG and PPG data, it is passed through hardware inbuilt filters and amplifiers. Final ADC raw ECG and PPG data is stored in an array.

This stored ECG data and PPG data are fetched by software programmer for data cleaning using implemented software filters. Once the ECG data and PPG data are cleaned without less noise, it is passed through peak detection algorithm using threshold. The detected ECG R peak locations and PPG peak locations are stored in array. PTT is calculated using ECG R peaks and PPG peaks. ECG heart rate is calculated using ECG R peaks. PPG heart rate is calculated using PPG peaks. BP is calculated using PTT, ECG heart rate and PPG heart rate.

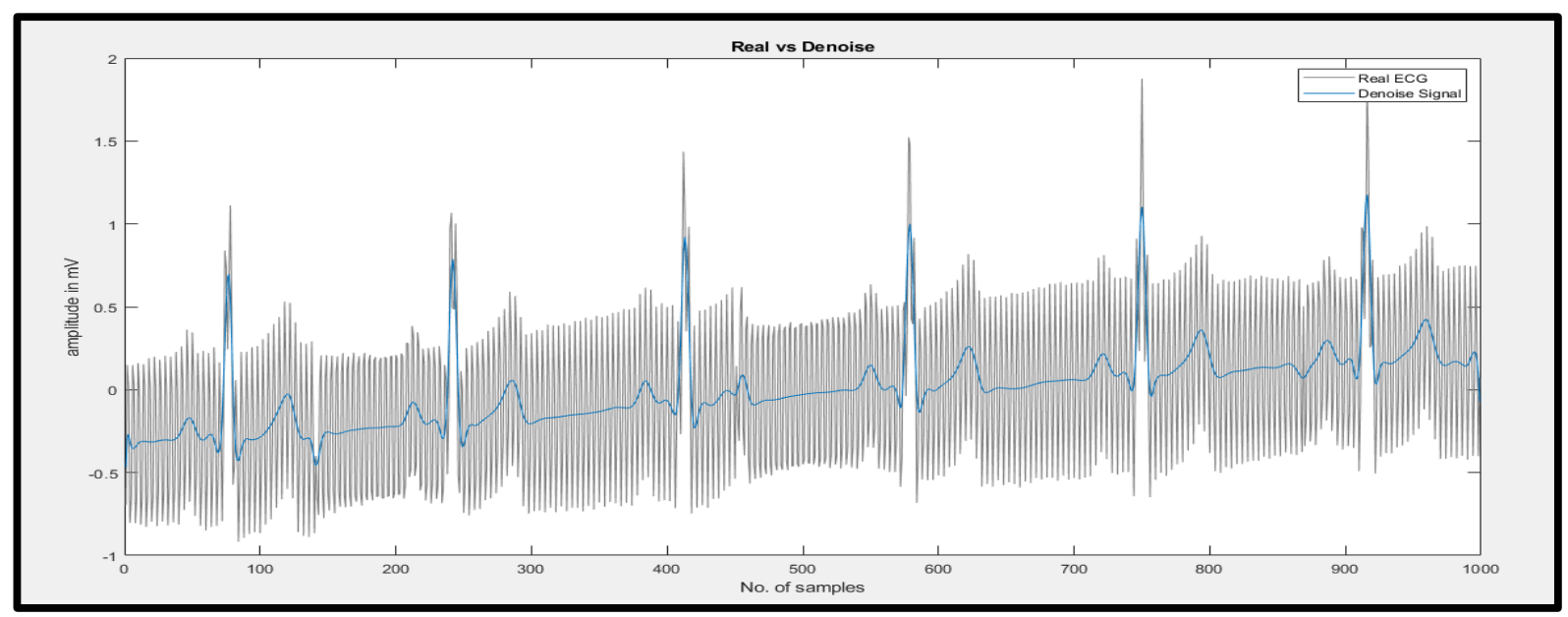

Fig 5(a). Raw vs Denoise ECG Data

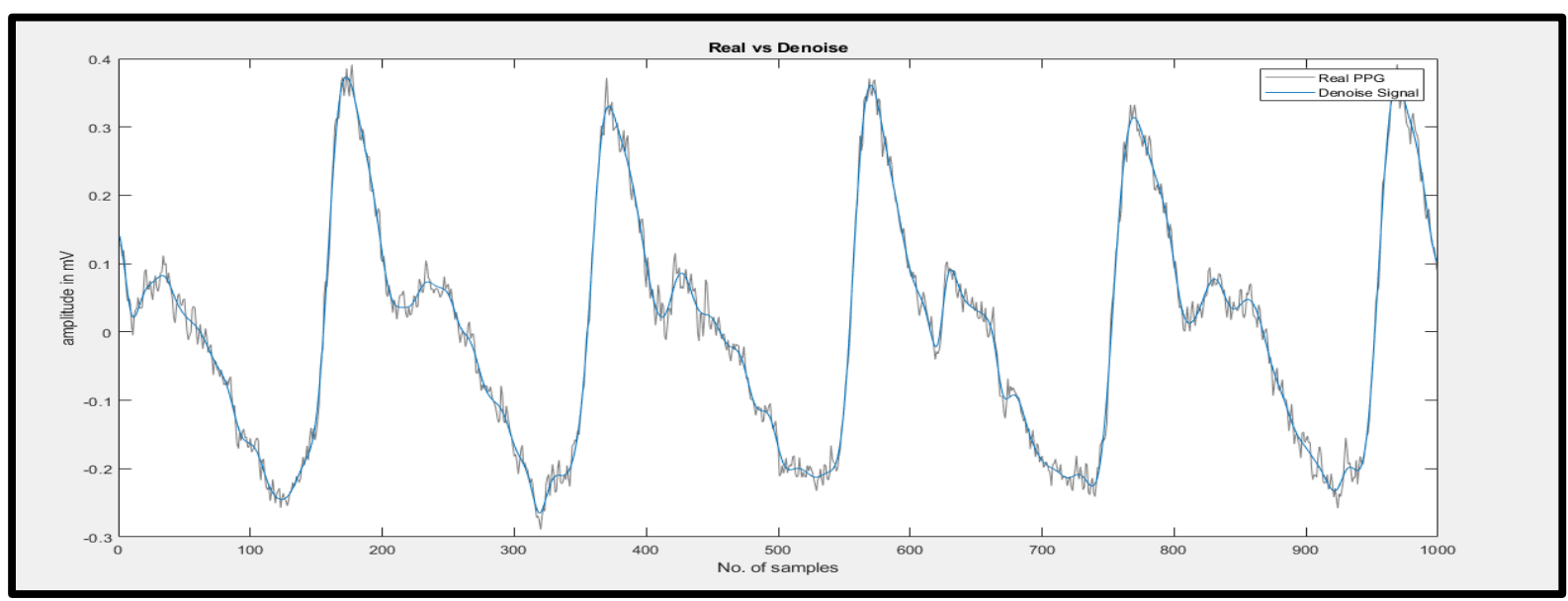

Fig 5(b). Raw vs Denoise PPG Data

\subsection{BP Estimation}

To read the ECG signal from three electrodes and PPG signal from sensor attached to wearable watch. After getting these values - find out Pulse Transit Time (PTT), which is time interval between ECG R-peak and PPG peak. ECG heartrate and PPG heartrate is calculated using ECG R peaks and PPG peak. Then using PTT, ECG heart rate and PPG heart rate - blood pressure can be calculated. In relation PTT is inversely proportional to Blood Pressure. 


\section{International Advanced Research Journal in Science, Engineering and Technology}

Vol. 8, Issue 8, August 2021

DOI: 10.17148/IARJSET.2021.8886

Error is calculated between watch reading and standard reading. It is given by,

SBP (Systolic Blood Pressure) Error in $\%=\frac{\text { (SBP from std device-SBP from watch device) }}{\text { (SBP from watch device) }}$

DBP (Diastolic Blood Pressure) Error in $\%=\frac{\text { (DBP from std device-DBP from watch device) }}{\text { (DBP from watch device) }}$

Table 1: Few Readings of BP with Error Percent

\begin{tabular}{|c|c|c|c|}
\hline Sl.No & $\begin{array}{l}\text { BP reading measured by Omron device } \\
\text { in } \mathrm{mm} \mathrm{Hg}\end{array}$ & $\begin{array}{l}\text { BP reading from wrist watch } \\
\text { device in } \mathrm{mm} \mathrm{Hg}\end{array}$ & Error in \% \\
\hline 1 & $\begin{array}{l}\text { SYS - } 159 \\
\text { DIA - } 102\end{array}$ & $\begin{array}{l}\text { SYS - } 155 \\
\text { DIA - } 98\end{array}$ & $\begin{array}{l}\text { SYS }-2.51 \% \\
\text { DIA }-3.92 \%\end{array}$ \\
\hline 2 & $\begin{array}{l}\text { SYS - } 114 \\
\text { DIA - } 70\end{array}$ & $\begin{array}{l}\text { SYS - } 114 \\
\text { DIA - } 72\end{array}$ & $\begin{array}{c}\text { SYS }-0 \% \\
\text { DIA }-2.85 \%\end{array}$ \\
\hline 3 & $\begin{array}{l}\text { SYS - } 109 \\
\text { DIA - } 68\end{array}$ & $\begin{array}{l}\text { SYS - } 110 \\
\text { DIA - } 70\end{array}$ & $\begin{array}{l}\text { SYS }-0.91 \% \\
\text { DIA }-2.94 \%\end{array}$ \\
\hline
\end{tabular}

Here, we have taken Omron cuff based blood pressure monitoring device as standard. To compare SBP and DBP reading from wrist watch device with Omron's SBP and DBP readings.

\section{CONCLUSION}

The method of blood pressure monitoring that is demonstrated in this project is cuffless and non-invasive method using ECG and PPG on wearable wrist watch has been proved to be more user friendly. It is portable. The blood pressure reading in the watch has around $92 \%$ accuracy and 7-8 \% Error percent with the test run we have done against the standard cuff based Omron blood pressure monitoring device. Here, whenever user wants to check their blood pressure, they can check using this wearable device. It also displays the reading on the watch and stores the BP reading in the server for further analysis

Further, BP algorithm should have to be tested with big data base with test run, to make it more robust. Hence this decreases the error percentage of the watch device against standard cuff based Omron blood pressure monitoring device. That will simultaneously increases the accuracy of the watch device.

\section{FUTURE SCOPE}

Integration of this paper with pattern recognition and machine learning. This will be another unexplored area where it can store BP reading pattern of each person and auto calibrate it to them. Machine learning will help to train the system to classify between normal BP and abnormal BP range. It will also train the system to classify various atrial fibrillation related to heart.

\section{REFERENCES}

1. Muskan Singla, Syed Azeemuddin, and Prasad Sistla, “Accurate Fiducial Point Detection Using Haar Wavelet for Beat-by-Beat Blood Pressure Estimation", IEEE, June 2020. 


\section{International Advanced Research Journal in Science, Engineering and Technology}

Vol. 8, Issue 8, August 2021

\section{DOI: 10.17148/IARJSET.2021.8886}

2. Remo Lazazzera, Yassir Belhaj and Guy Carrault, "A New Wearable Device for Blood Pressure Estimation Using Photoplethysmogram", MDPI-journal-sensors, June 2019.

3. Agnes S. Meidert and Bernd Saugel, “Techniques for Non-Invasive Monitoring of Arterial Blood Pressure”, Frontiers in Medicine, 2018.

4. Ramtanu Mukherjee, Sanchita Ghosh, Bharat Gupta and Tapas Chakravarty, "A Literature Review on Current and Proposed Technologies of Non-invasive Blood Pressure Measurement”, Telemedicine and E-Health, Mach 2018.

5. Manuja Sharma, Karinne Barbosa, Victor H, Devon Griggs, Tadesse Ghirmai, Sandeep K. Krishnan, Tzung K. Hsiai, Jung-Chih Chiao and Hung Cao, "Cuff-Less and Continuous Blood Pressure Monitoring: A Methodological Review”, MDPI-journal-technologies, May 2017.

6. Timothy B. Plante, Bruno Urrea, Zane T. MacFarlane, Roger S. Blumenthal, Edgar R. Miller, Lawrence J. Appel and Seth S. Martin, "Validation of the Instant Blood Pressure Smartphone App", JAMA Intern Med, 27 June 2016.

7. Ramakrishna Mukkamala and Jin-Oh Hahn, "Towards Ubiquitous Blood Pressure Monitoring via Pulse Transit Time: Theory and Practice", IEEE Trans Biomed Eng., 2016

8. Cao H, Kang B.J, Shung K.K and Hsiai T.K, "Electrical and Mechanical strategies to enable cardiac repair and regeneration", IEEE Rev. Biomed. Eng., 2015.

9. S. Noh, C. Yoon, E. Hyun, H.N. Yoon, T.J. Chung, K.S. Park and H.C. Kim, "Ferroelectret film-based patch-type sensor for continuous blood pressure monitoring", Electronics Letters, 30 ${ }^{\text {th }}$ January 2014.

10. S. Weber, D. Strommenger, U. Kertzscher and K. Affeld, "Continuous blood pressure measurement with ultrasound", Biomed Tech, 2012.

11. Laflamme M.A, Murry C.E, "Heart regeneration”, Nature, 2011.

12. Federico S. Cattivelli, Harinath Garudadri, "Noninvasive Cuffless Estimation of Blood Pressure from Pulse Arrival Time and Heart Rate with Adaptive Calibration", IEEE Tran,. pp. 114-119, 2009.

13. Jung Soo Kim, Ko Keun Kim, Hyun Jae Baek and Kwang Suk Park, "Effect of confounding factors on blood pressure estimation", IOP publishing, Pyhsiol. Meas.29, pp.615-624, 2008.

14. S Raju, M. S. Manikandan, B. Ramkumar, "Automatic method for Systolic PeakDetection", IEEE Trans. on Biomedical Signal Processing, pp. 41-46, 2014.

15. L. Peterson, R. Dripps, and G. Risman, "A method for recording the arterial pressure pulse and blood pressure in man," American Heart Journal, vol. 37, pp. 771-782, 1949. 